\title{
rintrojs: A Wrapper for the Intro.js Library
}

\section{Carl Ganz ${ }^{1}$}

DOI: $10.21105 /$ joss.00063

1 UCLA Center for Health Policy Research

\section{Software}

- Review ¿

- Repository ca

- Archive ${ }^{\top}$

\section{Licence}

Authors of JOSS papers retain copyright and release the work under a Creative Commons Attribution 4.0 International License (CC-BY).

\section{Summary}

Shiny (Chang et al. 2016) is an R package for developing web applications without HTML, CSS, or JavaScript. As Shiny increases in popularity so has the complexity of the Shiny apps built. In many instances, new-users will require instructions when they first use a Shiny application.

Intro.js (Mehrabani 2016) is a JavaScript library that helps integrate step-by-step introductions into websites. The rintrojs $\mathrm{R}$ package integrates Intro.js into Shiny, so that users can easily add instructions to their application without having to use JavaScript.

\section{References}

Chang, Winston, Joe Cheng, JJ Allaire, Yihui Xie, and Jonathan McPherson. 2016. Shiny: Web Application Framework for R. http://CRAN.R-project.org/package=shiny.

Mehrabani, Afshin. 2016. Intro.js: Better Introductions for Websites and Features with a Step-by-Step Guide for Your Projects. http://introjs.com. 